Revue Sciences/Lettres

Sciences / Lettres

$4 \mid 2016$

Baba Yaga en chair et en os

\title{
Une consommation marchandée : le cannibalisme comme dispositif d'échange et de transformation chez Baba Yaga
}

Jane Sinnett-Smith

\section{(2) OpenEdition \\ Journals}

Édition électronique

URL : http://journals.openedition.org/rsl/949

DOI : $10.4000 /$ rsl.949

ISSN : 2271-6246

Éditeur

Éditions Rue d'Ulm

Référence électronique

Jane Sinnett-Smith, « Une consommation marchandée : le cannibalisme comme dispositif d'échange et de transformation

chez Baba Yaga », Revue Sciences/Lettres [En ligne], 4 | 2016, mis en ligne le 16 janvier 2016, consulté le 20 avril 2019. URL : http://journals.openedition.org/rsl/949 ; DOI : 10.4000/rsl.949

Ce document a été généré automatiquement le 20 avril 2019.

(c) Revue Sciences/Lettres 


\section{Une consommation marchandée : le cannibalisme comme dispositif d'échange et de transformation chez Baba Yaga}

Jane Sinnett-Smith

1 Au même titre que son mortier bondissant et son isba à pattes de poule, le cannibalisme fait partie intégrante de l'image typique de Baba Yaga: elle apparaît dans l'imagination du public comme une sorcière féroce, qui dévore les enfants qui errent trop près de son domaine. Les contes cannibales de Baba Yaga se révèlent être un lieu exceptionnellement significatif pour les actes de transformation. Si la rencontre avec Baba Yaga est souvent considérée comme un moment d'initiation, une transformation du protagoniste d'enfant en adulte, le cannibalisme lui-même effectue des transformations complexes qui dérangent la frontière entre sujet et objet, humain et animal, intérieur et extérieur. Cet article met en question le rapport entre le cannibalisme et la transformation du héros, qu'il s'agisse d'une transformation de nature, de statut ou même d'un changement de forme par intervention magique. Nous soutenons que ce rapport se formule dans le contexte des échanges, des marchandises et des dons. Nous considérerons la manière dont un réseau complexe de rapports et d'échanges s'organise dans chaque conte entre Baba Yaga et le protagoniste, entre le protagoniste et la société, entre deux mondes distincts. Nous soutenons que le contact avec le cannibalisme laisse des traces importantes sur le protagoniste, ébranlant les distinctions que les contes s'efforcent d'établir.

2 À grands traits, les textes étudiés se rassemblent autour de deux motifs : celui de la fuite hors du four de la Baba Yaga, qui cherche à faire cuire et dévorer le héros, et celui de la gentille petite fille qui assure sa fuite en participant à une série d'échanges avec des créatures rencontrées pendant son aventure, ou avec la Baba Yaga elle-même.

3 Le premier groupe se compose de quatre variantes très proches : La Baba Yaga et Dégourdi, La Baba Yaga et Filiouchka, Ivachko et la sorcière, Tomassounet, et une version divergente 
Prince Daniel, mots de miel ${ }^{1}$. En substance, l'histoire raconte comment Baba Yaga enlève un petit garçon à sa famille pour le manger, et comment le héros la vainc et rentre sain et sauf au foyer. La clé de sa fuite est la ruse qu'il emploie : quand la complice de Baba Yaga, sa fille, essaie de le faire monter dans le four pour être cuit, il fait semblant de ne pas comprendre comment on y entre. Bien sûr, aussitôt que la fille y entre elle-même pour lui montrer comment faire, il ferme la porte et la laisse cuire. La Baba Yaga, à son insu, finit par manger sa propre fille, et le garçon s'échappe. Parfois, le héros réussit à duper et faire brûler Baba Yaga elle-même. On trouve aussi le motif du four dans Prince Daniel, mots de miel, encore qu'il soit très déformé : c'est la seule version de ce motif dans le recueil d'Afanassiev où le protagoniste est une fille. Le récit est une sorte de texte hybride, qui mélange plusieurs éléments de contes différents. L'héroïne, une princesse, en tentant d'échapper à son frère qui veut se marier avec elle, se retrouve dans la hutte de Baba Yaga. La fille de Baba Yaga, au lieu d'essayer de la faire cuire, l'aide à s'échapper, les deux filles conspirent pour tromper la Baba Yaga, et les deux prennent la fuite ensemble pendant que la Yaga est piégée dans son four.

4 Le deuxième groupe de contes inclut Les Oies Sauvages, La Baba Yaga et Vassilissa la Belle. La générosité de la protagoniste, voire son empressement à aider les étrangers qu'elle rencontre, est récompensée, car ces derniers l'aident en retour: dans les contes qui nous intéressent, ces rencontres l'aident à échapper à Baba Yaga. Dans Les Oies Sauvages, l'héroïne cherche à secourir son petit frère, qui a été enlevé par Baba Yaga à cause de sa négligence. En allant chez Baba Yaga elle rencontre une série d'objets animés, un four à pain, un pommier, une rivière de lait, qui la cachent de la fureur de Baba Yaga après lui avoir imposé certaines conditions. Dans le conte intitulé simplement La Baba Yaga, c'est la fille, envoyée dans l'isba de Baba Yaga par sa méchante marâtre, qui doit offrir des services aux serviteurs de Baba Yaga pour que ceux-ci la laissent fuir. Vassilissa la Belle n'entre pas facilement dans l'une ou l'autre des catégories de conte que nous avons établies², bien qu'il partage plusieurs éléments avec La Baba Yaga : Vassilissa est envoyée par sa marâtre, qui veut se débarrasser d'elle, chercher du feu chez la sorcière. Baba Yaga promet de l'aider si elle parvient à réaliser des tâches impossibles, tâches que Vassilissa accomplit avec l'aide d'une poupée magique, un don de sa défunte mère. Baba Yaga lui permet de partir avec du feu, feu qui, une fois porté chez Vassilissa, consume la méchante marâtre et les belles-sœurs. Vassilissa se marie avec un prince et vit heureuse.

\section{Les transformations}

5 La consommation est par sa nature même un acte qui donne lieu à des transformations de matière et de sens : la nourriture mangée est convertie de substance externe en élément interne, elle devient partie du corps du mangeur, intégrée à un système corporel plus large qu'elle ${ }^{3}$. La transformation subie est donc multiple : de l'extérieur vers l'intérieur, et d'un objet isolé en une composante d'un corps plus large. Lorsque cette consommation transformatrice devient aussi une consommation cannibale (avec toutes les connotations de transgression de tabou liées à l'anthropophagie), les transformations qu'elle effectue se multiplient de nouveau, et deviennent plus inquiétantes. En particulier, chez Baba Yaga, le cannibalisme menace la stabilité des frontières entre l'humanité et l'animalité, l'animé et l'inanimé. Ainsi, en plus des motifs de transfert extérieur-intérieur, isolementcommunauté, nous identifions plusieurs types de transformation subis par les victimes du cannibalisme de Baba Yaga. 


\subsection{Humain-animal}

Le conte Vassilissa la Belle décrit comment la sorcière mange des humains « comme des poulets ${ }^{4} »$. L'acte de manger une personne réévalue le statut de cette personne. Un des privilèges tacites de l'humanité est d'occuper uniquement la position de mangeur, de prédateur, et jamais celle de mangé, de proie. En refusant ce privilège à sa victime, le cannibale effectue un glissement troublant de catégorie et déstabilise le statut privilégié de l'humanités. Le conte de Vassilissa suggère que la consommation de l'humain, sa transformation en proie, est incompréhensible sans le recours à une métaphore d'animalité. Si un élément essentiel de l'humanité est le fait de ne pas être mangé, le seul moyen de comprendre ce changement de statut est d'imaginer la victime humaine comme un animal. Le cannibalisme de Baba Yaga initie donc une transformation à deux niveaux, de statut et de nature : la victime est transformée d'être privilégié en créature vulnérable et d'être humain en animal. Cette animalisation de la victime rappelle de manière inquiétante que l'humanité n'est qu'une catégorie supérieure d'animal. Mais elle est loin d'être la transformation la plus dramatique dans les contes cannibales.

\subsection{Sujet-objet}

7 Plusieurs contes narrent comment le désir cannibale de Baba Yaga transforme sa victime d'actant, de sujet conscient, en objet inanimé. Dans le conte La Baba Yaga, celle-ci proclame à propos du protagoniste qu'elle veut «en faire [son] déjeuner ${ }^{6}$ ». De manière similaire, dans Prince Daniel, mots de miel, la fille de Baba Yaga lui explique qu'elle n'a pas retenu un groupe de passantes pour les manger parce que celles-ci étaient trop "vieilles ", et donc pas au goût de sa mère, «trop dures pour [ses] dents ». De la même manière, dans le conte de Vassilissa, on perçoit la victime comme un animal car on n'arrive pas à concevoir l'acte de dévorer un être humain : ici Baba Yaga ne reconnaît pas la subjectivité de ses victimes désignées. Elle les perçoit seulement du point de vue gastronomique, comme des repas ou de la nourriture. On trouve l'exemple le plus dramatique de procédés selon lequel le cannibalisme transforme la victime en objet inanimé dans La Baba Yaga et Filiouchka. Cela vaut la peine d'examiner l'épisode entier où la fille de Baba Yaga pénètre dans le four et y est rôtie ${ }^{7}$.

Pas comme cela, Filiouchka! dit la fille de la Yaga brune. - Et comment alors ? J'sais pas, moi! - Attends, je vais te montrer!», et elle s'allongea comme il faut sur la pelle. Filiouchka, [...] la saisit en un tournemain et la lança dans le poêle [...] [il] ouvrit la porte et sortit sa victime, rôtie. Il l'enduisit de graisse, la posa sur un plat qu'il recouvrit d'un linge, et rangea le tout dans le coffre [...] la Yaga brune arrive, fouille le coffre, en retire le rôti ${ }^{8}$.

Ce passage raconte un processus de transformation linguistique extraordinaire. Au début la fille participe au conte comme un sujet parlant, capable d'action et de pensée. Si elle n'est pas nommée dans cette version du conte (elle l'est dans des variantes), elle est au moins identifiée clairement comme une personne («la fille...»). Sa transformation en victime est aussi une transformation en objet. Il y a un glissement de vocabulaire, de « fille » en « victime » puis simplement en « rôti ». Elle devient un simple ingrédient dans une recette, objet inerte qui sert au plaisir d'un autre. 


\subsection{Mangé-Mangeur}

9 Il est intéressant de noter que si l'on considère des représentations du cannibalisme plus larges, surtout les rites rapportés par des anthropologues, ou même l'acte de cannibalisme symbolique qu'est l'Eucharistie 9 , on découvre que le cannibalisme a souvent pour effet de transformer le cannibale lui-même, qu'il s'agisse d'une sorte d'initiation à la maturité ou de l'incorporation dans une communauté. Mais malgré les transformations éprouvées par ses victimes, Baba Yaga elle-même ne semble pas changer de nature au fil de son cannibalisme, elle n'est pas sujette aux transformations effectuées. Nous suggérons que le cannibalisme est si constitutif du personnage de Baba Yaga, à la fois changeant et immuable ${ }^{10}$, que les contes ne peuvent concevoir une métamorphose ni de son caractère ni de sa nature. Qu'elle soit menaçante ou bienveillante, elle est toujours Baba Yaga, et impossible à transformer. Cela ne signifie pas que Baba Yaga n'est pas du tout touchée par les transformations impliquées par la consommation. En dévorant la victime, elle l'incorpore dans son propre corps : son corps est constitué par ceux de ses victimes. Sa consommation trouble la frontière entre le corps qui mange et le corps qui est mangé. En effet, la section suivante de cet article revient plus précisément sur l'idée que, au cours du conte, les identités de Baba Yaga et de sa victime semblent se brouiller et se ressembler.

\section{Les échanges}

10 Il est important de noter que malgré les multiples références au cannibalisme de Baba Yaga, les contes ne racontent la réalisation de ses appétits cannibales que peu fréquemment ${ }^{11}$. En général, la menace cannibale de Baba Yaga demeure à l'état de menace : elle constitue un défi que doit vaincre le protagoniste. La victoire contre la sorcière et son cannibalisme est assurée par la participation du héros aux processus d'échange - en fait, chaque conte dans le recueil d'Afanassiev qui montrent le cannibalisme de Baba Yaga contient une sorte d'échange, qu'il s'agisse de mercantilisme pur ou d'un échange plutôt symbolique. Le motif de l'échange n'est pas propre aux contes du cannibalisme, mais son omniprésence dans ces contes le rend particulièrement remarquable.

11 La nature transformatrice du cannibalisme est seulement exacerbée par son implication dans les processus d'échange. La forme de la transformation effectuée est bien sûr différente et ne vise plus seulement la victime (ou disons la victime désignée), mais s'étend au monde qui l'entoure. Le «type » de transformation le plus basique effectué dans ces contes - voire peut-être dans tous contes de fées - est le développement du protagoniste d'enfant en adulte ${ }^{12}$. Cette section examine la manière dont la menace du cannibalisme stimule cette transformation fondamentale, et comment l'engagement des protagonistes dans des réseaux d'échanges peut à la fois les distinguer de Baba Yaga et aggraver leur ressemblance à la sorcière.

\subsection{Le cannibalisme comme punition : échanges avec Baba Yaga}

12 La Baba Yaga semble rarement déclencher l'échange d'elle-même. Dans Vassilissa la Belle et Ivachko et la sorcière, elle utilise son cannibalisme comme une menace afin d'assurer que 
l'on respecte les termes des échanges qu'elle propose. Elle déclare à Vassilissa: «Tu vas travailler chez moi quelque temps : si je suis contente, je te donnerai du feu, sinon, je te mangerai ${ }^{13}$ !». L'issue de l'échange détermine la position que Vassilissa occupe : si elle arrive à accomplir les tâches que Baba Yaga lui a assignées, elle peut participer à l'échange comme celle qui rend un service et qui reçoit le don de feu. Si elle échoue, elle devient simplement l'objet échangé, ou plutôt pris, par Baba Yaga. Cet échange est clairement déséquilibré : les conditions de Baba Yaga (de séparer le froment du son, les graines de pavot de la terre) sont impossibles. Baba Yaga perturbe la logique de l'échange réciproque en instaurant un rapport injuste qui ne favorise qu'elle : elle essaie de s'établir comme double bénéficiaire du travail de Vassilissa, et de son corps, sans rien donner.

Cet échange déséquilibré est encore plus marqué dans Ivachko et la sorcière. Baba Yaga déclare au forgeron qu'elle le mangera s'il ne lui donne une voix douce qui lui permettra d'attirer le petit garçon qu'elle veut manger, et des dents de fer pour le chasser: «Forgeron, forgeron! Forge-moi une voix aussi douce que celle de la mère d'Ivachko, sinon je vais te manger !», «Forgeron, forgeron! Forge-moi des dents de fer, sinon je te mange $!^{14} »$. Le cannibalisme fonctionne donc comme une punition à laquelle la réalisation de l'échange permettra d'échapper. Le forgeron est payé pour le service qu'il rend à Baba Yaga non par un bénéfice, un profit concret, mais par un manque (le fait que Baba Yaga s'abstienne de le dévorer). En fait, il faut interroger la possibilité de qualifier cet épisode de vrai échange: Baba Yaga reçoit quelque chose du forgeron, mais elle ne donne rien. Bien qu'ailleurs dans les contes Baba Yaga fonctionne très souvent comme donneuse de conseils ou de cadeaux magiques, sous son aspect cannibale elle reste en dehors des systèmes d'échanges, elle refuse d'y participer, de donner autant que de recevoir. Au lieu de l'échange réciproque, elle se meut dans un système d'exigence et de prise. Elle reste à part des structures d'échange qui unissent la société, elle ne participe pas à une communautét ${ }^{15}$.

\subsection{Les dons récompensés : échanges avec la société}

Si ces deux contes mettent l'accent sur le caractère isolé et fermé de Baba Yaga et de son monde, les contes mettant en scène la gentille petite fille et ses échanges au gré des rencontres contrastent fortement avec les contes évoqués ci-dessus. Le type d'échange réalisé dans ces contes est au contraire explicitement réciproque : la fille donne, et son don est rémunéré par l'aide du bénéficiaire. Les Oies Sauvages, un conte hanté par la menace cannibale de Baba Yaga, illustre l'importance de ce partage mutuel du rôle de donateur et de récepteur. La première fois que l'héroïne, en route vers l'isba de Baba Yaga pour récupérer son frère, rencontre les autres participants à l'échange, elle refuse de coopérer. Chaque fois l'être rencontré dit qu'il lui révélera où les oies emportent son frère si elle remplit les termes de l'échange qu'il propose: le four à pain demande qu'elle mange son pain, le pommier une de ses pommes, la rivière de lait un peu du sirop qui forme ses rives $^{16}$. Elle exige qu'ils lui fournissent les informations qu'elle cherche gratuitement, rejetant leurs conditions et ainsi refusant le contre-don. Elle se comporte, autrement dit, comme la Baba Yaga elle-même. Ce n'est qu'au cours de la deuxième rencontre, de retour de la hutte et fuyant Baba Yaga, que la fillette accède aux requêtes de ses interlocuteurs et, ce faisant, garantit sa survie. 
15 Le conte relate donc comment la fille apprend à participer aux systèmes d'échange. L'œuvre fondamentale de Marcel Mauss, Essai sur le don (1925), met en valeur l'importance de l'échange mutuel pour lier et unir une communauté ${ }^{17}$. Mauss décrit le réseau complexe d'obligation mis en place par l'échange des dons: l'obligation simultanée qu'ont les participants de donner, recevoir, et rendre ${ }^{18}$. Ce conte semble dramatiser la centralité de cet échange réciproque et commun. La rencontre de la protagoniste avec des objets anthropomorphes variés incarne les trois pôles d'obligation de Mauss dans un seul moment d'échange. La fille reconnaît son devoir de recevoir du four, du pommier, de la rivière, le don qu'ils veulent lui donner. Sa consommation est donc à la fois un don de leur part (elle reçoit de la nourriture) et de sa part (elle remplit leur demande). Chaque partie utilise la notion de la dette, c'est-à-dire l'obligation de rendre après avoir reçu un don, pour aboutir à leurs fins. La menace du cannibalisme transforme l'héroïne d'actant égoïste, qui veut prendre sans donner et sans recevoir, en participant actif qui comprend les dettes qu'elle doit à la société autour d'elle.

Nous suggérons donc que ce récit enseigne la manière d'appartenir à la société. La fillette apprend comment rejeter le mode de consommation égoïste incarnée par Baba Yaga, comment éliminer une ressemblance entre elle et la sorcière. En revanche, elle se conçoit comme membre d'un groupe social auquel elle contribue et duquel elle reçoit dans un système d'obligations, mais aussi, pour nous éloigner un peu des termes de Mauss, de profit mutuel. Finalement la fille et ceux qu'elle rencontre exploitent le réseau d'obligation pour obtenir ce dont ils ont envie. Il est important de noter que ce qui la sauve est l'acte de manger, de partager de la nourriture, un geste puissant d'unification qui forge la communauté19. L'acte d'échanger de la nourriture, un service, de l'aide, crée des liens qui unissent la fille et les créatures rencontrées au sein d'une même communauté.

Si le conte décrit la transformation d'un individu en membre d'une communauté soudée par des échanges mutuels, il déclare aussi la supériorité des valeurs de ce mode de vie. Il établit deux modèles rivaux de comportement, l'égoïsme de la prise et le partage des dons mutuels. Ce n'est que l'initiation réussie du protagoniste au système d'échanges mutuels qui assure sa survie, une légitimation claire de ce mode de vie commun. Le texte déclare que ce ne sont pas seulement les échanges qui construisent la société, mais que seule la participation à ces échanges permet l'accès à cette société. Toutefois, le fait de ne pas adhérer à ces valeurs implique une absorption dans le monde de Baba Yaga - non seulement à travers une incorporation littérale par la consommation, mais aussi à travers un système d'appropriation qui ne serait pas gouverné par les obligations mutuelles de l'échange.

\subsection{Un échange de système}

La rencontre de Baba Yaga et du protagoniste est donc une rencontre entre le monde de la victime et le monde de Baba Yaga. Dans Vassilissa la Belle cette rencontre de mondes met en scène une confrontation non seulement des deux systèmes (de partage ou de prise) mais de deux types de consommation. La consommation est au centre du texte: Vassilissa s'assure de n'être pas dévorée par Baba Yaga en donnant de la nourriture à sa poupée magique, qui l'aide à remplir les termes de l'échange de Baba Yaga. Vassilissa substitue à un type de dévoration - le risque d'être dévorée par Baba Yaga - une autre dévoration - celle des repas par sa poupée. Fait significatif, elle change la nature de la 
consommation effectuée. Baba Yaga se livre à une consommation essentiellement solitaire, égoïste : Vassilissa lui sert une nourriture suffisante pour dix personnes, qu'elle mange en totalité, ne laissant à Vassilissa que quelques miettes ${ }^{20}$. En revanche, l'alimentation de la poupée fait partie d'un échange réciproque: Vassilissa donne de la nourriture pour recevoir quelque chose de retour. La dévoration égoïste perpétrée par Baba Yaga est remplacée par un système de réciprocité, le mangeur transformé de simple profiteur en acteur participant à l'échange de dons/contre-dons.

Ce qui est particulièrement intéressant est le fait que Vassilissa se prive souvent des repas pour alimenter sa poupée ${ }^{21}$. Bien qu'elle évite le cannibalisme littéral de Baba Yaga, elle semble s'engager dans une forme de cannibalisme sublimé, voire symbolique : elle donne à la poupée la nourriture qui aurait dû la nourrir, qui aurait dû être incorporée dans son propre corps, un don symbolique de sa propre chair $^{22}$. Ce don suggère peut-être une part de sacrifice dans la notion de l'échange, mais il faut noter qu'il est loin d'être sans intérêt personnel. Vassilissa ne se sacrifie pas sans attendre une récompense concrète de celui qui reçoit. En fait, Mauss note que dans les systèmes d'échange de dons, bien qu'un objet puisse être donné sous l'apparence d'un cadeau, le don porte toujours des implications d'obligations et d'exigences de remboursement ${ }^{23}$.

Le conte La Baba Yaga confirme l'aspect intéressé des échanges entamés par le protagoniste. Comme le suppose le topos de la gentille petite fille, la participation de l'héroïne aux échanges avec la communauté assure le succès de sa fuite. Cependant, c'est l'héroïne elle-même qui propose ces échanges aux serviteurs de Baba Yaga : elle donne un foulard à la servante pour mouiller les bûches du feu sur lequel elle devrait être cuisinée, du jambon au chat pour qu'il ne lui arrache pas les yeux, du pain aux chiens pour qu'ils ne la mettent pas en pièces, de l'huile au portail pour la laisser passer sans grincer, un ruban au bouleau pour qu'il ne l'égratigne pas ${ }^{24}$. Le don de la nourriture, ou d'un autre type de ressource essentielle, occupe le devant de la scène: comme Vassilissa, l'héroïne de ce conte change le système égoïste de consommation de Baba Yaga pour son propre système de consommation partagée. Lorsque Baba Yaga interroge ses serviteurs pour découvrir pourquoi ils l'ont trahie, ils se plaignent qu'elle ne leur a jamais payé leurs services, tandis que la fillette était prête à établir un système de contrepartie.

21 Cette plainte met l'accent sur le besoin de réciprocité pour gérer une société, mais aussi sur les attentes des participants à cette société. Si Les Oies Sauvages enseigne que l'on ne peut pas recevoir sans donner, $\mathrm{La}$ Baba Yaga suggère que personne ne donne sans attendre une récompense. Même la générosité de l'héroïne n'existe pas sans obligations : dans la même phrase où elle donne un foulard à la bonne comme un " cadeau », elle lui présente la contrepartie de ce don (empêcher le progrès du feu pour la cuisiner). Elle ne donne pas simplement par pure bonté de cœur, mais dans l'attente que ses cadeaux soient rémunérés. C'est-à-dire que les échanges dans lesquels elle s'engage ressemblent moins à un partage commun égal qu'à un acte marchand, une vente et un achat de services. Le système d'échanges qui gouverne la société est un système qui cache des traces de mercantilisme : la rencontre avec le cannibalisme de Baba Yaga enseigne au protagoniste, et aux autres membres de sa communauté, la valeur commercialisable de leurs services.

En gérant les marchandises avec les serviteurs de Baba Yaga, la fille effectue une transformation non seulement de la nature du système de l'échange, mais aussi de son propre statut dans ce système. Elle se convertit de victime de cannibalisme et objet d'échange (elle a été envoyée par sa belle-mère à Baba Yaga pour être dévorée) en vainqueur de Baba Yaga et actrice de l'échange, donatrice et receveuse. En changeant sa 
position dans le schéma objet-donneur-bénéficiant, elle échappe à la menace immédiate du cannibalisme. Cependant, à travers les obligations qu'elle impose aux créatures qui l'entourent ${ }^{25}$, à travers l'aisance avec laquelle les serviteurs de Baba Yaga la servent elle à la place de leur maîtresse originelle, le texte semble entretenir l'idée d'une ressemblance entre Baba Yaga et sa victime d'autrefois.

\subsection{Une transformation de victime : ressemblance victime-Baba Yaga}

23 Les récits qui mettent en scène le motif du four examinent cette possible similarité entre Baba Yaga et sa victime plus en détail. La substitution de la victime nous présente un des cas rares où le conte narre la réalisation du cannibalisme de Baba Yaga : ignorante de la ruse du héros, Baba Yaga mange sa propre fille. En transformant la victime, le conte arrive à transformer la nature de la consommation racontée. Si la dévoration du héros violait les exigences de la morale du conte où le Bien doit vaincre le Mal, la dévoration de la fille complice maintient le cannibalisme dans ce système moral ${ }^{26}$.

Qui plus est, le fait que Baba Yaga ne mange que sa propre fille renferme sa consommation transgressive dans sa propre communauté familiale, et assure qu'elle ne corrompt pas le reste de la société humaine. Dans les exemples les plus extrêmes du conte, La Baba Yaga et Dégourdi, Prince Daniel, c'est la Baba Yaga elle-même qui est enfermée et qui périt dans le four: sa menace est radicalement éliminée, elle ne trouble plus la société27.

Elle effectue donc un échange de place avec sa victime, qui est elle-même métamorphosée de victime en vainqueur, d'enfant innocent en protagoniste habile. Cette progression de position vulnérable en position de pouvoir affirme l'intégration du héros dans la société. Elle confirme l'idée de la rencontre avec Baba Yaga comme d'une sorte d'acte d'initiation. La plupart des protagonistes sont des petits garçons, et Andreas Johns considère le refus du héros d'entrer dans le four comme une rupture avec la mère (Baba Yaga), un refus de rentrer dans son ventre (le four) ${ }^{28}$. Nous ajoutons que le héros quitte son statut d'objet de prise en participant à l'échange, l'aventure raconte donc l'entrée du protagoniste dans la société adulte, sa prise de conscience qu'il peut participer à des systèmes d'échange qui gouvernent cette société. Néanmoins, le fait qu'il rôtit Baba Yaga et ses filles avec une telle jubilation suggère qu'il peut agir comme l'analogue de Baba Yaga : il adopte le rôle de Baba Yaga avec une alacrité qui dément la séparation des mondes de Baba Yaga et du protagoniste et l'effort d'affirmer les valeurs de la société à laquelle le protagoniste appartient en éliminant le cannibalisme de Baba Yaga ou en l'enfermant dans un système familial clos.

En effet, le conte de Prince Daniel suggère que l'exclusion des traits indésirables incarnés par Baba Yaga est loin d'être sans ambiguïté. Au lieu de duper et brûler la fille de Baba Yaga, l'héroïne forge une communauté avec elle - la fille dérobe l'héroïne aux yeux de Baba Yaga en la transformant en aiguille, elles enferment Baba Yaga dans le four ensemble, et la fille finit par épouser le frère de l'héroïne ${ }^{29}$. Ce parent proche de Baba Yaga, même si elle aide le héros, ne parvient pas à se purifier des indices inquiétants du cannibalisme de sa mère : en transformant l'héroïne en aiguille elle lui fait subir la même métamorphose en objet inanimé que la Baba Yaga cannibale voulait effectuer. L'intégration de cet enfant de Baba Yaga dans la société par le mariage suggère donc que la frontière claire que les contes essayent de construire entre le système égoïste, 
antisocial et hors la société de la cannibale Baba Yaga et le système réciproque d'échange et de communauté du protagoniste n'est pas sûre et infranchissable. Le système social de partage communautaire abrite toujours la possibilité de l'égoïsme du cannibalisme. Encore plus inquiétant : la fille de Baba Yaga ressemble à la sœur si parfaitement qu'elles sont indistinctes, ce qui suggère le manque essentiel de différence entre la souillure de Baba Yaga que le conte essaie d'exclure et les valeurs qu'il soutient.

\section{Conclusions}

Le cannibalisme de Baba Yaga peut être une force très utile pour forger la société. Les échanges auxquels les protagonistes doivent participer pour y échapper leur permettent l'entrée dans une communauté et définissent les limites de cette communauté. Une démarcation claire est construite entre le monde de Baba Yaga, où l'on prend sans payer et où l'on ne partage pas, et le monde du héros, où les échanges sont réciproques et produisent un profit mutuel. La construction de ce système d'échanges suggère à la fois l'idéalisme et le mercantilisme: il enseigne la responsabilité envers la communauté (l'obligation de recevoir et de rendre) et aussi un sentiment de droit (l'attente qu'à tour de rôle on sera récompensé). Cependant le cannibalisme est essentiellement un acte qui transgresse des frontières, entre l'humanité et l'animalité, l'animé et l'inanimé, le comportement acceptable et ce qui doit être exclu. Nous soutenons que malgré leurs efforts, les contes ne réussissent pas à se purger complètement des implications du cannibalisme de Baba Yaga, ne parviennent pas à ériger une frontière définitive entre le monde de Baba Yaga et le nôtre. L'ambiguïté qui en résulte rappelle que cela peut toujours resurgir dans l'individu et dans la communauté et appelle à une nécessaire vigilance. En effet, au cours des échanges dans lesquels les protagonistes s'engagent pour échapper aux griffes de Baba Yaga, ils peuvent finir par lui ressembler plutôt que se différencier de son mode de vie. Au bout du compte, l'échange entre ces mondes qui est le sens de la rencontre entre Baba Yaga et le protagoniste déstabilise toujours la tentative de les séparer.

\section{BIBLIOGRAPHIE}

Afanassiev, Alexandre Nikolaiévitch, Contes populaires russes, t. I, trad. Lise Gruel-Apert, Paris, Imago, 2009.

Arens, W., The Man-eating Myth : Anthropology and Anthropophagy, New York, Oxford University Press, 1979.

Avramescu, Catalin, An Intellectual History of Cannibalism, Princeton, Princeton University Press, 2011.

Balina, Marina, Goscilo, Helena, et Lipovetsky, Mark, (dir.), Politicising Magic : An Anthology of Russian and Soviet Fairy Tales, Evanston, Illinois, Northwestern University Press, 2005. 
Guest, Kristen (dir.), Eating Their Words : Cannibalism and the Boundaries of Cultural Identity, Albany, State University of New York Press, 2001.

Johns, Andreas, Baba Yaga : The Ambiguous Mother and Witch of the Russian Folktale, New York, Peter Lang, 2004.

Kilgour, Maggie, From Communion to Cannibalism : An Anatomy of Metaphors of Incorporation, Princeton, Princeton University Press, 1990.

Mauss, Marcel, The Gift : The form and reason for exchange in archaic societies, trad. W. D. Halls, Londres, Routledge Classics, 2002.

Propp, Vladimir, Les Racines historiques du conte merveilleux, Paris, Gallimard, 1983.

Reeves Sanday, Peggy, Divine Hunger : Cannibalism as a Cultural System, Cambridge, Cambridge University Press, 1986.

Singer, Eliot A., « The narrative functions of food in Afanas'ev's fairy tales », Semiotica, 57, 1985, p. 339-368.

Sinnett-Smith, Jane, « Dents d'acier et enfants rôtis : Baba Yaga et le cannibalisme », in LETAP (collectif), Baba Yaga. Workshop., Paris, Éditions l'Imprimante, 2014, p. 48-59.

Steel, Karl, How to Make a Human : Animals and Violence in the Middle Ages, Columbus, Ohio State University Press, 2011.

\section{NOTES}

1. Tous les textes étudiés se trouvent dans A. N. Afanassiev, Contes populaires russes (cité CPR infra), Tome I.

2. On a identifié ce conte exceptionnellement long et détaillé comme une création plutôt littéraire que folklorique. Voir A. Johns, Baba Yaga: The Ambiguous Mother and Witch of the Russian Folktale, p. 117.

3. M. Kilgour, From Communion to Cannibalism: An Anatomy of Metaphors of Incorporation, p. 4.

4. A. N. Afanassiev, CPR, p. 141.

5. Karl Steel, How to Make a Human: Animals and Violence in the Middle Ages, p. 124.

6. A. N. Afanassiev, CPR, p. 137.

7. Nous examinons cet épisode également dans J. Sinnett-Smith, « Dents d'acier et enfants rôtis : Baba Yaga et le cannibalisme », p. 49-50.

8. A. N. Afanassiev, CPR, p. 153.

9. Pour une discussion du rapport entre la communion et le cannibalisme, voir M. Kilgour, op. cit., p. 15-18 ou W. Arens, The Man-eating Myth : Anthropology and Anthropophagy, p. 16, p. 160.

10. M. Balina, H. Goscilo et M. Lipovetsky, (dir.), Politicising Magic: An Anthology of Russian and Soviet Fairy Tales, p. 13.

11. J. Sinnett-Smith, op. cit., p. 49.

12. V. Propp, a notamment étudié Baba Yaga comme un personnage central dans le «rite d'initiation » subi par le protagoniste, voir Les Racines historiques du conte merveilleux, p. 142.

13. A. N. Afanassiev, CPR, p. 142.

14. Ibid., p. 156-157.

15. J. Sinnett-Smith, op. cit., p. 57.

16. A. N. Afanassiev, CPR, p. 161.

17. M. Mauss, traduit par W. D. Halls, The Gift: The form and reason for exchange in archaic societies, p. 6 . 
18. Ibid., p. 50.

19. E. Singer, «The narrative functions of food in Afanas'ev's fairy tales », p. 362.

20. «Vassilissa [...] se mit à retirer du four plats et rôtis : il y en avait bien là pour dix personnes [...] La vielle but et mangea tout. » A. N. Afanassiev, CPR, p. 143.

21. «Écoute-moi, Vassilissa [dit la mère] [...] Je meurs et, avec ma bénédiction, je te laisse cette poupée [...] Chaque fois que tu seras en peine, offre-lui de la nourriture et demande-lui conseil. » Ibid., p. 140.

22. Nous étudions le rapport entre le cannibalisme de Baba Yaga et les consommations alternatives proposées par ses victimes d'un autre point de vue dans J. Sinnett-Smith, op. cit., p. 53-54.

23. M. Mauss, op. cit., p. 3-4.

24. A. N. Afanassiev, CPR, p. 136-137.

25. Ainsi que celles qu'ils lui imposent dans Les Oies Sauvages et Vassilissa la Belle.

26. J. Sinnett-Smith, op. cit., p. 50.

27. A. N. Afanassiev, CPR, p. 152 et p. 166.

28. A. Johns, op. cit., p. 99.

29. A. N. Afanassiev, CPR, p. 165-167.

\section{RÉSUMÉS}

Cet article examine la manière dont la consommation cannibale de Baba Yaga fonctionne au sein des rapports divers établis entre la sorcière et ses victimes. Il suggère que le cannibalisme est le foyer d'un réseau d'échanges entre Baba Yaga et la victime, entre la victime et les autres membres de la société, entre les mondes différents. Ces échanges provoquent une série de transformations qui établissent les limites de ce qui peut être incorporé à la société, et de ce qui doit être exclu.

This article examines the ways in which Baba Yaga's cannibal consumption works within a context of the various relationships established between the witch and her victims. It argues that cannibalism is home to a network of exchanges between Baba Yaga and her victim, between her victim and the other members of their society, between different worlds. These exchanges provoke a series of transformations that establish the limits of what can be incorporated into society, and what must be excluded.

\section{INDEX}

Mots-clés : cannibalisme, échange, don

Keywords : cannibalism, exchange, gift 


\section{AUTEUR}

\section{JANE SINNETT-SMITH}

Jane Sinnett-Smith est étudiante à University College London. Elle se spécialise dans la littérature française médiévale et prépare une thèse sur l'utilisation des reliques comme objets de foi, de merveille ou de préoccupation dans la littérature séculaire.

jane.sinnett-smith.14@ucl.ac.uk 